\title{
Gastric collision tumor: Case report of a rare adenocarcinoma and a typical carcinoid tumor
}

\author{
BETÜL ÜNAL ${ }^{1}$, GÜLSÜM ÖZLEM ELPEK ${ }^{1}$, TEKINALP GELEN $^{1}$, ALIHAN GÜRKAN ${ }^{2}$ and BÜLENT YILDIRIM $^{3}$ \\ Departments of ${ }^{1}$ Pathology, ${ }^{2}$ General Surgery and ${ }^{3}$ Internal Medicine, \\ Akdeniz University Medical School, Antalya 07070, Turkey
}

Received December 9, 2012; Accepted February 27, 2013

DOI: $10.3892 / \mathrm{ol} .2013 .1313$

\begin{abstract}
We report a case with features of gastric adenocarcinoma colliding with a typical carcinoid component. A 51-year-old female was admitted to the Department of Internal Medicine with complaints of epigastric pain. On physical examination of the patient there was significant epigastric tenderness and the CA19-9 level was higher than the normal titer value. An upper gastrointestinal endoscopy showed an ulcerated polypoid mass located on the cardiac region of the stomach. Pathological and immunohistochemical findings diagnosed as a collision tumor comprising both adenocarcinoma and carcinoid tumor. Metastasis of adenocarcinoma was found in 7 perigastric lymph nodes, while metastasis of the carcinoid was not detected. The admixture of neoplastic endocrine and nonendocrine cells, have been found infrequently in gastric tumors. The mixed tumors can be further classified into composite tumors that show an admixture of two histological components with histological transitions and collision tumors where the two components are not intermixed In general it is not easy to morphologically distinguish a collision tumor, from composite tumor. Microscopically, hematoxylin and eosin-stained tissue sections from two different areas of the mass revealed two different types of tumor; an intestinal type adenocarcinoma and a carcinoid tumor. We report a case with features of adenocarcinoma colliding with a typical carcinoid component, along with a review of the literature.
\end{abstract}

\section{Introduction}

The admixture of neoplastic endocrine and non-endocrine cells have been identified infrequently in gastric tumors. For such lesions, four distinct categories are distinguished according to the tissue morphological features: carci-

Correspondence to: Dr Betül Ünal, Department of Pathology, Akdeniz University Medical School, Dumlupınar Street, Antalya 07070, Turkey

E-mail: betulunalmd@hotmail.com

Key words: collision tumor, gastric adenocarcinoma, carcinoid nomas with interspersed endocrine cells, carcinoids with interspersed non-endocrine cells, mixed tumors and amphicrine tumors. Mixed tumors may be further classified into composite tumors, which exhibit an admixture of two histological components with histological transitions, and collision tumors, where the two components are not intermixed (1). While carcinoma with interspersed endocrine cells is the most frequent, the remaining tumors have rarely been reported in the stomach, with collision tumor being particularly unusual (1).

In the present study, we report a case with features of adenocarcinoma colliding with a typical carcinoid component, along with a review of the literature. The study was approved by the Ethics Committee of Akdeniz Unversity Medical Faculty, Antalya, Turkey. Written informed consent was obtained from the patient.

\section{Case report}

A 51-year-old female was admitted to the Department of Internal Medicine of Akdeniz University Medical School, Antalya, Turkey, with complaints of epigastric pain. On physical examination there was significant epigastric tenderness. The biochemical analyses of blood and urine were within normal ranges. While the CEA level was not elevated, the CA19-9 level was higher than the normal titer value $(82 \mathrm{U} / \mathrm{ml}$; reference range, 0-40 U/ml). An upper gastrointestinal endoscopy showed an ulcerated polypoid mass located on the cardia. The histopathological examination of multiple biopsies from this lesion revealed a gastric adenocarcinoma. The patient underwent a total gastrectomy with lymph node dissection.

Macroscopic examination revealed an ulcerated polypoid mass measuring $2.5 \times 1.5 \times 2 \mathrm{~cm}$. The dissected surface showed a yellow-white area surrounding the area of the ulcer. On closer inspection, a more yellow region $(1 \mathrm{~cm})$ was observed below the normal location of the gastric mucosal folds. This region and the ulcerated yellow area were abutted to each other (Fig. 1).

Microscopically, hematoxylin and eosin-stained tissue sections from two different areas of the mass showed two different types of tumor. One was a moderately differentiated intestinal type adenocarcinoma and the other was a tumor composed of a relatively uniform population of small cells with organoid, trabecular or focally solid patterns, suggesting neuroendocrine cell proliferation (Fig. 2). These 
Table I. Summary of previous studies on gastric collision tumor composed of an adenocarcinoma and a carcinoid tumor.

\begin{tabular}{lcll}
\hline & Age & & \\
Authors (Ref.) & (years) & Gender & Location \\
\hline Yamashina M et al (11) & 50 & Male & Corpus \\
Chodankar CM et al (10) & 69 & Female & Corpus \\
Morishita Y et al (9) & 49 & Male & Corpus \\
Corsi A et al (8) & 72 & Male & Unknown \\
Camuñas Mohinelo FA et al (7) & 66 & Male & Cardia \\
Olinici CD et al (6) & 68 & Male & Corpus \\
Morishita Y et al (5) & 84 & Female & Cardia \\
Jayaraman A et al (4) & 48 & Male & Antrum \\
Doggui MH et al (3) & 55 & Male & Fundus \\
Mróz A et al (2) & 56 & Male & Corpus \\
Present case & 51 & Female & Cardia \\
\hline
\end{tabular}

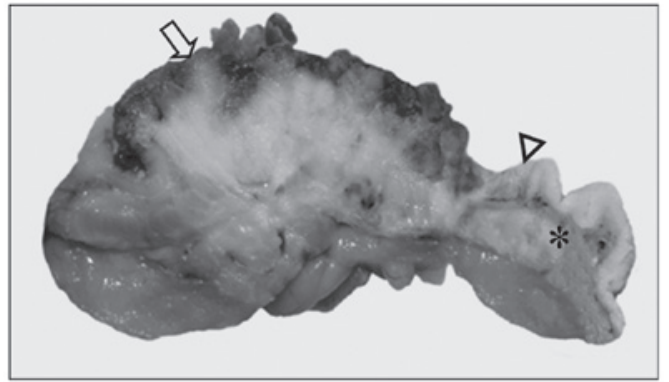

Figure 1. Macroscopic findings of the resected specimen. The two lesions were detected macroscopically; a large ulcerated polypoid mass (arrow) and a yellow lesion (asterisk) which was observed underneath the normal location of the gastric mucosa (arrowhead).

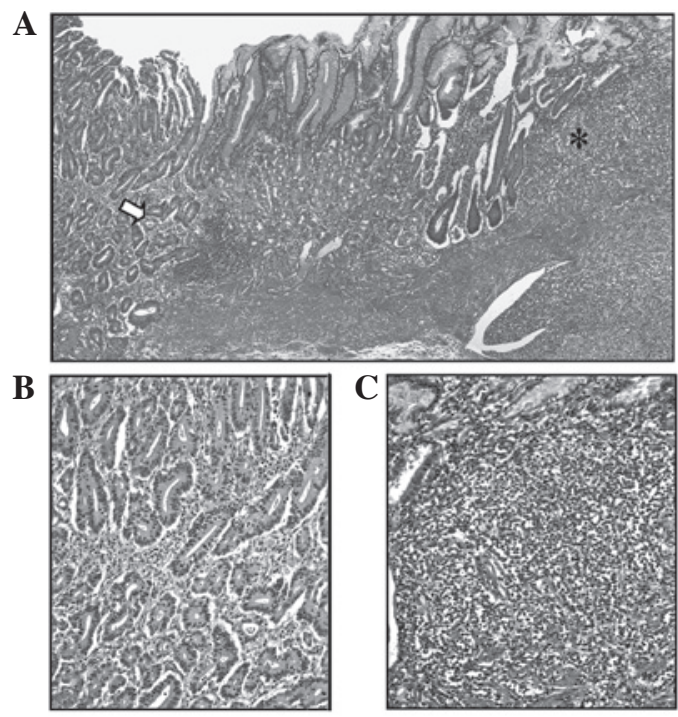

Figure 2. Histopathological findings of the collision tumor (hematoxylin and eosin staining). (A) Large polypoid tumor revealed moderately differentiated adenocarcinoma (arrow) and another tumor composed of small cells (asterisk). The two tumors were colliding in the gastric cardia and were clearly separated. Magnification, x100. (B) Larger view of the adenocarcinoma forming gland-like structures (arrow in A). Magnification, x200. (C) Larger view of the carcinoid tumor composed of a uniform population of small cells with solid patterns (asterisk in A). Magnification, x200.
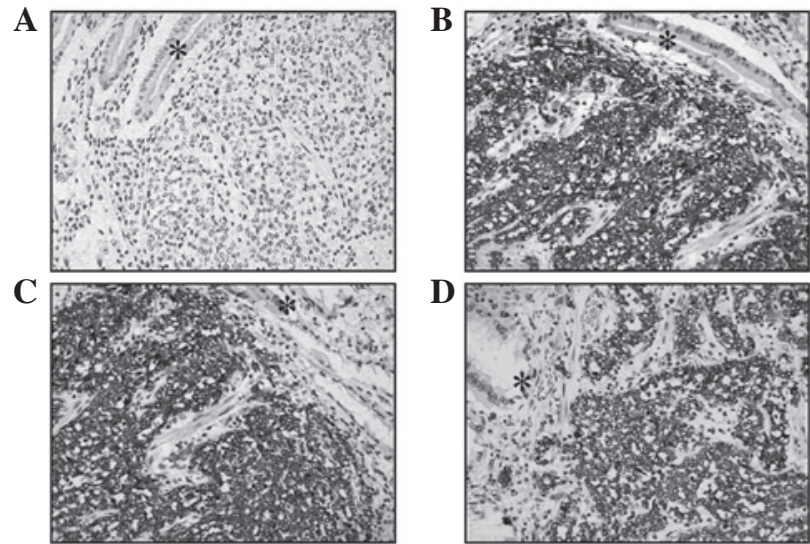

$\mathbf{E}$
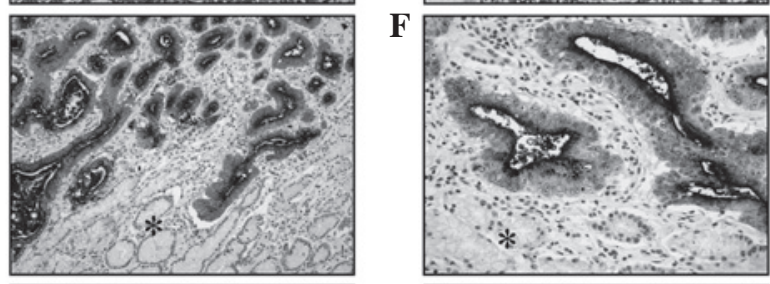

G
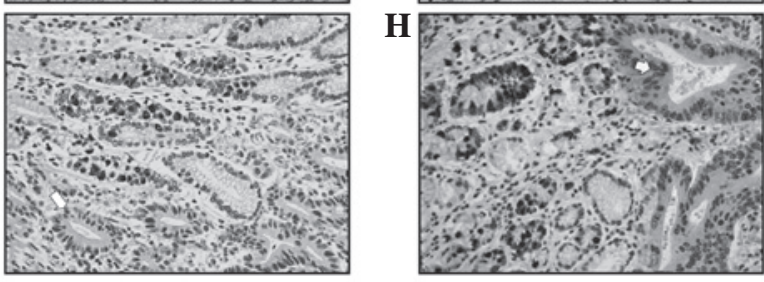

Figure 3. Immunohistochemical findings (avidin biotin peroxidase method counterstained with hematoxylin). (A) Tumor cells which formed the carcinoid component did not stain for CEA. (B-D) Cells markedly expressed NSE, chromogranin A and synaptophysin, respectively. The normal gastric mucosa is indicated by an asterisk. (E and F) Tumor cells of the adenocarcinoma stained for CEA, but not for $(\mathrm{G})$ chromogranin A and $(\mathrm{H})$ synaptophysin. Neuroendocrine cells of gastric glands stained for $(\mathrm{G})$ chromogranin A and (H) synaptophysin. Arrows indicate tumor areas. (A-H) Magnification, x400.

cells exhibited a granular cytoplasm and an indistinct cytoplasmic border. Their nuclei had homogenous chromatin with indistinct nucleoli. Mitosis was infrequent (1/10 hpf) and necrosis was not observed. Immunohistochemical analysis showed that these cells had strong positivity for neuroendocrine markers (synaptophysin, chromogranin A and NSE) and this section of the tumor was diagnosed as a typical carcinoid tumor (Fig. 3). By contrast, adenocarcinoma cells expressed only CEA (Fig. 3). As the two tumors were distinctly separated from each other and no merging of tissue components was noted at the interface of the growth, the final diagnosis was a collision tumor composed of an adenocarcinoma and a carcinoid tumor of the stomach. The two tumors invaded the subserosal layer. While lymphatic permeation by the adenocarcinoma was noted, the carcinoid component was negative for lymphatic permeation. Vascular invasion was not observed for either component. Metastasis of the adenocarcinoma was identified in 7 perigastric lymph nodes among the 12 dissected lymph nodes, while metastasis of the carcinoid tumor was not detected.

Clinical course. The patient succumbed to the tumor progression five months after surgery. 


\section{Discussion}

The present case involved a 51-year-old female with an ulcerated polypoid mass located at the cardiac region of the stomach. According to the pathological and immunohistochemical findings, the mass was diagnosed as a collision tumor comprising an adenocarcinoma and a carcinoid tumor. Although the presence of adenocarcinoma or carcinoid tumor individually is not notable, this collision tumor with two histopathological types in the stomach is only the eleventh case in the current literature. The patient in the present case report is younger than those reported in previous cases (mean, 61.7 years) and contrasts the frequent male predilection for this type of lesion (male/female, 4:1), demonstrating that collision tumors of this type are not only limited to older age groups or to males (Table I) (2-11).

As shown in the present case, gastric collision tumors comprising an adenocarcinoma and a carcinoid tumor are usually solitary lesions $(2,4-11)$. The majority of previously reported tumors were located in the corpus $(2,6,9-11)$. However, two previous cases were localized at the cardia (similar to the present case); one case was identified in the antrum and another in the fundus, suggesting that this tumor type may be encountered in different locations in the stomach (Table I) (3-5,7).

In general it is difficult to morphologically distinguish a collision tumor from a composite tumor. However, in the present case, the tumor comprised two components with different histopathological and immunohistochemical features. The two constituents were abutting each other without histological transition between them. Although metastasis of a composite tumor comprises both of the tissue components, metastasis of a collision tumor includes only a single tissue component (1). In our case report, lymph node metastases were monomorphic and had an adenocarcinoma component. Another problem in the correct diagnosis of collision tumors comprising an adenocarcinoma and a carcinoid tumor is that a diagnosis based on endoscopic biopsy may depend on the sampled site of the tumor. In these cases, if the biopsy specimen revealed only the carcinoid component, treatment and surgical intervention may be different.

Although pathogenic factors that contribute to the development of an adenocarcinoma or a carcinoid tumor alone have been extensively described, the pathogenesis of collision tumors comprising adenocarcinoma and carcinoid tumor is unclear. For instance, diet, genetics and infection with Helicobacter pylori may contribute to the development of gastric adenocarcinoma. Pernicious anemia and gastric atrophy may be a contributing factor for the development of a gastric carcinoid tumor (5). Alternatively, it has been postulated that carcinoid tumors are able to produce substances with a growth-promoting effect, which may account for the occurrence of a secondary tumor in the vicinity (12). In the present case, pernicious anemia and atrophic gastritis were not observed and the fasting serum gastrin value was not elevated. These tumors are considered to have arisen independently from multipotential epithelial stem cells and primitive neuroendocrine cells. In a previous report which carried out allelotyping analysis to study the genetic profiles of the endocrine and exocrine components of six mixed tumors of the gut, Furlan et al observed clonal divergence in a collision tumor, which was composed of a well-differentiated endocrine carcinoma associated with an adenocarcinoma (13). This finding confirms the existence of double tumors which grow next to each other coincidentally, yet exhibit different histogenesis and different tumorigenetic pathways.

The prognosis of this rare entity is unclear; however, from the few known cases it appears that the adenocarcinoma impacts more heavily on prognosis $(1,5)$. The patient in this case succumbed to the tumor five months after gastric resection due to widespread liver metastasis of the adenocarcinoma.

In conclusion, the present case is the eleventh gastric collision tumor of its type, composed of an adenocarcinoma and a carcinoid tumor and this confirms their presence at this location. As further cases of this tumor type are reported, the clinicopathological properties and pathogenesis of this entity are likely to be revealed in more detail.

\section{Acknowledgements}

This study was supported by Akdeniz University Scientific Projects Unit.

\section{References}

1. Graeme-Cook F: Neuroendocrine tumors of the GI tract and appendix. In: Surgical Pathology of the GI Tract, Liver, Biliary tract, and Pancreas. Odze RD, Goldblum JR and Crawford JM (eds). 1st edition. WB Saunders, Philadelphia, PA, pp491-496, 2004.

2. Mróz A, Kiedrowski M, Malinowska M and Sopyło R: Collision tumour of the stomach - adenocarcinoma and neuroendocrine carcinoma: case report and review of the literature. Pol J Pathol 60: 94-97, 2009.

3. Doggui MH, Ben Yaghlène L, Hefaiedh R, Bouguassas W, Mestiri A and Dellagi K: A gastric collision tumor composed of adenocarcinoma and gastrinoma: case report. Tunis Med 86: 755-757, 2008.

4. Jayaraman A, Ramesh S, Jeyasingh R and Bagyalakshmi KR: Gastric collision tumour - a case report. Indian J Pathol Microbiol 48: 264-265, 2005.

5. Morishita Y, Sugitani M, Sheikh A, Nemoto N, Fujii M and Takayama T: Collision tumor of the stomach: a rare case of an adenocarcinoma and carcinoid tumor. Arch Pathol Lab Med 129: 407-409, 2005.

6. Olinici CD, Crişan D and Racu I: Synchronous gastric adenocarcinoma and carcinoid. Rom J Gastroenterol 13: 135-137, 2004.

7. Camuñas Mohinelo FA, Melgar Requena P, Martínez Zaragoza J, et al: Gastric collision tumor with osseous metaplasia. Rev Esp Enferm Dig 89: 317-319, 1997 (In Spanish).

8. Corsi A and Bosman C: Adenocarcinoma and atypical carcinoid: morphological study of a gastric collision-type tumour in the carcinoma-carcinoid spectrum. Ital J Gastroenterol 27: 303-308, 1995.

9. Morishita Y, Tanaka T, Kato K, et al: Gastric collision tumor (carcinoid and adenocarcinoma) with gastritis cystica profunda. Arch Pathol Lab Med 115: 1006-1010, 1991.

10. Chodankar CM, Pandit SP, Motiwale SS and Deodhar KP: Collision tumour of stomach. Indian J Gastroenterol 8: 297-298, 1989.

11. Yamashina M and Flinner RA: Concurrent occurrence of adenocarcinoma and carcinoid tumor of the stomach: a composite tumor or collision tumors? Am J Clin Pathol 83: 233-236, 1985.

12. Gerstle JT, Kauffman GL and Koltun WA: The incidence, management, and outcome of patients with gastrointestinal carcinoids and second primary malignancies. J Am Coll Surg 180: 427-432, 1995.

13. Furlan D, Cerutti R, Genasetti A, et al: Microallelotyping defines the monoclonal or the polyclonal origin of mixed and collision endocrine-exocrine tumors of the gut. Lab Invest 83: 963-971, 2003. 